\title{
ENDOMORPHISM RINGS OF REDUCED COMPLETE TORSION-FREE MODULES OVER COMPLETE DISCRETE VALUATION RINGS
}

\author{
WOLFGANG LIEBERT ${ }^{1}$
}

\begin{abstract}
The purpose of this paper is to give necessary and sufficient conditions for an abstract ring to be isomorphic to the endomorphism ring of a reduced complete torsion-free module over a complete discrete valuation ring.
\end{abstract}

In [3] we have characterized the endomorphism rings of reduced complete torsion-free modules over (not necessarily commutative) complete discrete valuation rings. By generalizing the Harrison-Matlis duality of [1] and [4] to the noncommutative case, this work simultaneously characterizes endomorphism rings of divisible torsion modules. The model for our main result was Wolfson's beautiful characterization of the ring of all linear transformations of a vector space over a division ring in [5]. The purpose of this note is to show how Wolfson's theorem can be used directly for the characterization of the endomorphism rings of these modules.

WOLFSON'S THEOREM. Let $E$ be $a$ ring and $E_{0}$ its right socle. Then the following are equivalent:

I. $E$ is isomorphic to the ring of all linear transformations of a vector space over a division ring.

II. (1) $E_{0}$ is not a zero-ring, and is contained in every nonzero two-sided ideal of $E$.

(2) If $L$ is a left ideal of $E$ which is annihilated on the right only by zero, then $E_{0} \subset L$.

(3) The sum of two left (right) annihilators is a left (right) annihilator.

(4) E possesses an identity element.

Definition. A ring which satisfies condition I or II in the theorem above is said to be a Wolfson ring.

Received by the editors February $28,1972$.

AMS (MOS) subject classifications (1970). Primary 16A48, 16A42, 16A64; Secondary 16A80, 20K20.

Key words and phrases. Endomorphism rings, Wolfson rings, reduced complete torsion-free modules, complete discrete valuation rings, $J$-adic topology.

${ }^{1}$ This research was supported by NSF grant GP-28379.

(C) American Mathematical Society 1973 
Let $E$ be a ring. We endow $E$ with the $J$-adic topology by taking the powers $J(E)^{n}(n=0,1,2, \cdots)$ of its Jacobson radical $J(E)$ as a basis of neighborhoods of 0 ; this makes $E$ a topological ring.

THEOREM. Let $E$ be a ring with Jacobson radical $J(E)$. Then the following three properties are equivalent:

I. $E$ is isomorphic to the endomorphism ring of a divisible torsion module over a complete discrete valuation ring.

II. $E$ is isomorphic to the endomorphism ring of a reduced complete torsion-free module over a complete discrete valuation ring.

III. (1) $E$ is Hausdorff and complete in its J-adic topology.

(2) $E / J(E)$ is a Wolfson ring.

(3) $J(E)=p E=E p$, where $p$ is either zero or a nonzero-divisor of $E$.

Proof. The equivalence of I and II and the implication II $\rightarrow$ III were proved in Theorems 5.5 and 5.6 of [3]. It remains to show that III implies II.

Assume now that (1)-(3) of III are valid. If $p=0$ in (3), then there is nothing to prove in view of Wolfson's theorem. We shall henceforth assume that $p \neq 0$. First, we wish to prove that $E$ possesses an identity element. Put $\bar{E}=E / J(E)$, and for $\alpha \in E$ let $\bar{\alpha}$ denote the natural image of $\alpha$ in $\bar{E}$. We know that $\bar{E}$, being a Wolfson ring, has an identity $\bar{f}$. Since $E$ is Hausdorff and complete in its $J$-adic topology, idempotents modulo $J(E)$ can be lifted (see e.g. [2, pp. 124-125]). So we may assume that $f^{2}=f \in E$. Naturally we claim that $f$ is the identity of $E$. To see that, write $E=f E \oplus N$ where $N=\{x-f x \mid x \in E\}$. Then the right ideal $N$ must be contained in $J(E)$. In fact, $N$ must be the zero ideal. Assume $0 \neq y \in N$. Then, by hypothesis (3), there exists $z \neq 0$ in $E$ such that $y=z p$. It also follows from (3) that we can write $z=z^{\prime} p^{n}$ for suitable $n \in Z$ and $z^{\prime} \notin J(E)$. Clearly $z^{\prime} \notin N$. Also $z^{\prime} \notin f E$ since otherwise $y=z^{\prime} p^{n+1} \in f E$. We can therefore write $z^{\prime}=a+b$ with $0 \neq a \in f E$ and $0 \neq b \in N$. Now $y=z^{\prime} p^{n+1}=a p^{n+1}+b p^{n+1}$ with $a p^{n+1} \in f E$ and $b p^{n+1} \in N$. Hence $a p^{n+1}=0$, which contradicts our hypothesis that $p$ is not a zero-divisor. It follows that $N=0$, which means that $f$ is a left identity for the ring $E=f E$. By symmetry, $f$ is also a right identity for $E$. Hence $f=1$ is the identity for the ring $E$.

Next we wish to establish an important property of the so-called minimal nonradical right ideals of $E$. As in [3] we call a right ideal $H$ of $E$ minimal nonradical if $H$ itself is not contained in $J(E)$, but every right ideal of $E$ which is properly contained in $H$ lies in $J(E)$. Let us show the existence of minimal nonradical right ideals in $E$. Since $\bar{E}$ is a Wolfson ring, there exists a minimal right ideal $\bar{e} \bar{E}$ in $\bar{E}$ with $\bar{e}^{2}=\bar{e} \in \bar{E}$. We can choose $e \in E$ such that $e^{2}=e$ because idempotents modulo $J(E)$ can be 
lifted. Then the isomorphism

$$
e E e / J(e E e)=e E e / J(E) \cap e E e \cong e E e+J(E) / J(E)=\bar{e} \bar{E} \bar{e}
$$

shows that $e E e / J(e E e)$ is a division ring. Consequently, $e E$ is a minimal nonradical right ideal in $E$, by virtue of Lemma 9.1 in [3]. Moreover, the technique of lifting idempotents can be used to show that every minimal nonradical right ideal of $E$ has the form $e E$ with $e^{2}=e \in E$. This latter fact, however, also follows from hypothesis (3) and the Hausdorffness of $E$, as was demonstrated in Lemma 7.1 of [3].

Suppose now that $B$ is a two-sided ideal of $E$ which is not contained in $J(E)$. Let $e E$ be any minimal nonradical right ideal of $E, e^{2}=e \in E$. Since $\bar{E}$ is a Wolfson ring, it follows that $\bar{B}$ contains the right socle of $\bar{E}$. Now $\bar{e} \bar{E}=\bar{e} \bar{E}$ is a minimal right ideal in $\bar{E}$. Therefore, $\bar{e} \in \bar{B}$, and we can find $\gamma \in J(E)$ such that $e+\gamma \in B$. Since $1+\gamma$ is a unit in $E$, we see that

$$
e=e(1+\gamma)(1+\gamma)^{-1}=(e+e \gamma)(1+\gamma)^{-1}=e(e+\gamma)(1+\gamma)^{-1} \in B
$$

Hence $e E \subseteq B$. Accordingly, if $E_{0}$ denotes the sum of all minimal nonradical right ideals of $E$, we may sum up our conclusions thus far by saying that

(4) $E_{0}$ is not a zero-ring and is contained in every nonradical two-sided ideal of $E$.

It was shown in [3, pp. 167-168] that (1) and (3) together with property (4) imply the existence of a reduced complete torsion-free module $H$ over a complete discrete valuation ring $R$ such that $E$ is isomorphic to a subring of the ring $E_{R}(H)$ of all $R$-endomorphisms of $H$. More precisely, $H=e E$ and $R=e E e$, where $e E$ is a minimal nonradical right ideal of $E$. The action of the $R$-endomorphism induced by the element $\alpha \in E$ is simply right multiplication of the elements of $H$ by $\alpha$ within the ring $E$.

It remains to show that $E$ is isomorphic to the full ring $E_{R}(H)$. Since $H$ is a minimal nonradical right ideal of $E$, the right ideal $\bar{H}=\bar{e} \bar{E}$ must be minimal in $\bar{E}$. Moreover, $\bar{H}=H / p H$ where $p$ denotes the unique prime element of the ring $R$ (see [3, pp. 167-168]). Of course, $\bar{E}$ induces a certain ring of $\bar{e} \bar{E} \bar{e}$-endomorphisms of the $\bar{e} \bar{E} \bar{e}$-vector space $\bar{e} \bar{E}$, again by right multiplication. This must be the full $\bar{e} \bar{E} \bar{e}$-endomorphism ring, according to the proof of Wolfson's theorem in [5]. It follows at once from Theorem 5.6 in [3] that a subring $S$ of $E_{R}(H)$ is dense in $E_{R}(H)$ in the $J$-adic topology if and only if $S$ induces the full $R / p R$-endomorphism ring in $H / p H$. Therefore, our hypothesis (1) implies that $E \cong E_{R}(H)$, and now the proof is complete. 


\section{REFERENCES}

1. D. K. Harrison, Infinite abelian groups and homological methods, Ann. of Math. (2) 69 (1959), 366-391. MR 21 \#3481.

2. I. Kaplansky, Fields and rings, Univ. of Chicago Press, Chicago, Ill., 1969. MR 42 \#4345.

3. W. Liebert, Characterization of the endomorphism rings of divisible torsion modules and reduced complete torsion-free modules over complete discrete valuation rings, Pacific J. Math. 37 (1971), 141-170.

4. E. Matlis, Cotorsion modules, Mem. Amer. Math. Soc. No. 49 (1964). MR 31 \#2283.

5. K. G. Wolfson, An ideal-theoretic characterization of the ring of all linear transformations, Amer. J. Math. 75 (1953), 358-386. MR 14, 718.

Department of Mathematics, New Mexico State University, Las Cruces, New MEXICO 88001

Current address: Mathematisches Institut, Technische Universität, Arcisstrasse 21, 8 München, West Germany 